\title{
COMAROSTAPHYLIS MACVAUGHII STAT. NOV., UNA ERICACEAE MEXICANA, ELEVADA A NIVEL DE ESPECIE ${ }^{1}$
}

\author{
Luz Ma. Gonzalez Villarreal \\ Instituto de Botánica \\ Universidad de Guadalajara \\ Apartado Postal 139 \\ Zapopan, Jalisco 45110
}

\section{RESUMEN}

Se propone Comarostaphylis macvaughii stat. nov., a base de Comarostaphylis discolor (Hooker) Diggs subsp. macvaughii Diggs, ya que esta planta presenta notables diferencias morfológicas con respecto a las demás subespecies descritas.

\section{ABSTRACT}

Comarostaphylis macvaughii stat. nov., is proposed on the basis of Comarostaphylis discolor (Hooker) Diggs subsp. macvaughii Diggs, in view of remarkable morphological differences observed in this plant, as compared with the remaining subespecies.

Durante el estudio taxonómico de la familia Ericaceae para el Estado de Jalisco, al examinar las cuatro subespecies de Comarostaphylis discolor reconocidas por Diggs (1988), se encontraron diferencias notables que sugieren elevar al rango de especie a $C$. discolor subsp. macvaughii.

Se trabajó con ejemplares vivos y materiales de herbario; dentro de este último, se examinaron más de 50 especímenes pertenecientes a $C$. discolor (Hooker) Diggs subsp. discolor, C. discolor (Hooker) Diggs subsp. rupestris, C. discolor (Hooker) Diggs subsp. macvaughii y $C$. discolor (Hooker) Diggs subsp. manantlensis, depositados en ENCB, IBUG, IEB, MEXU, NY, WIS y XAL.

Todas estas subespecies están representadas en las montañas de Jalisco, excepto la subsp. rupestris que, según Diggs (1986), está restringida a Michoacán.

Comarostaphylis macvaughii (Diggs) González V., stat. nov. (Fig. 1).

Comarostaphylis discolor (Hooker) Diggs subsp. macvaughii Diggs, Bull. Torrey Bot.

Club 115 (3): 204. 1988. Tipo: MEXICO. Jalisco: Mountains 10-11 km N of Tapalpa, rd. to Chiquilistlán, pine forest $\mathrm{nr}$. summits, $2300-2350 \mathrm{~m}$, shrubs to $10 \mathrm{~cm}$ high, $30-31$ January 1975 (fl, fr). McVaugh 25967 (holotipo, MICH; foto del holotipo, IBUG!).

\footnotetext{
'Trabajo apoyado por la Universidad de Guadalajara mediante convenios 85-01/263 y 86/MB/0331/01/ 0260.
} 


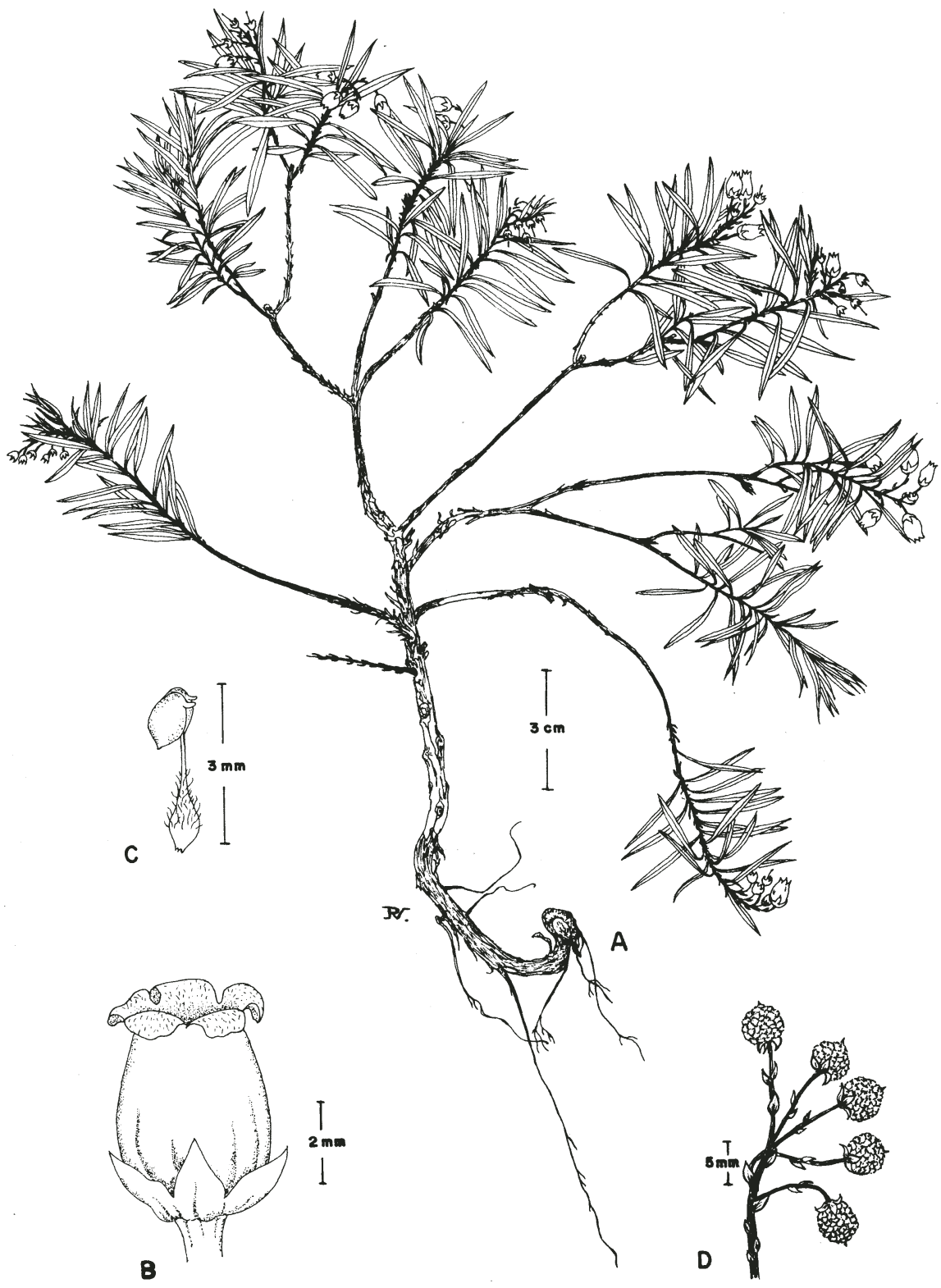

Fig. 1 Comarostaphylis macvaughii (Gónzalez Villarreal 2598). A. Hábito; B. Flor; C. Detalle del estambre; D. Infrutescencia. 
Arbusto pequeño erecto, cespitoso, rizomatoso, de (6-) 10-15 (-25) cm de alto, corteza exfoliante en pequeñas placas delgadas de color grisáceo a café-rojizo, ramillas glabras; hojas subsésiles, coriáceas, de color verde claro, por lo general con manchas rojizas, lineares a linearlanceoladas, de (1-) 1.5-2 (-4) cm de largo, de (1-) 1.5-2 (-5) mm de ancho (excepcionalmente de $6 \mathrm{~cm}$ de largo por $7 \mathrm{~mm}$ de ancho), margen entero, muy rara vez con unos cuantos dientes (sobre todo en renuevos vigorosos), ligeramente revoluto, ápice agudo a acuminado, mucronado, base cuneada a aguda; haz y envés glabros, aunque en la juventud con una diminuta pubescencia confinada a la nervadura central y a la base de la hoja; inflorescencias en racimos, raquis glabros de $1.5-2(-2.5) \mathrm{cm}$ de largo, a veces flores solitarias, brácteas carinadas a naviculares, agudas, de (1.2-) 1.5-3 (-5) $\mathrm{mm}$ de largo, de 1 (-2) $\mathrm{mm}$ de ancho, pedicelos de 4$7(-8) \mathrm{mm}$ de largo, glabros, bractéolas en la parte media del pedicelo, lanceoladas, de (1-) 1.5 (-2.3) mm de largo; cáliz rojizo con los lóbulos triangulares a ovado-triangulares, de 1-1.5 (-1.7) $\mathrm{mm}$ de largo, de (1-) $1.2(-1.7) \mathrm{mm}$ de ancho, ápice agudo con un pequeño mucrón, ciliados; corola rosada, de (4-) 4.5-5.5 (-6) mm de largo, de 3.5-4.5 (-5) mm de ancho, glabra por fuera, internamente pilosa, sus lóbulos de (0.8-) $1.2(-1.6) \mathrm{mm}$ de largo, de (1-) $1.5(-1.8) \mathrm{mm}$ de ancho; estambres 10, de 2.5-3.5 mm de largo, filamentos de (2-) $2.5(-3.5) \mathrm{mm}$ de largo, ensanchados de la base, vilosos, anteras glabras, ovoides, de (1.1-) $1.3(-1.5) \mathrm{mm}$ de largo, de 0.7-0.8 (-1) $\mathrm{mm}$ de ancho, con 2 apéndices cortos de 0.4-0.5 (-0.6) mm de largo; ovario glabro, estilo columnar de (2.5-) 3-3.5 mm de largo, glabro; fruto drupáceo, globoso, inicialmente rojizo, negro al madurar, de (3-) 4 (-4.5) mm de largo, de (3-) 4 (-5) mm de diámetro, superficie papilosa; semillas de 2-4 $\mathrm{mm}$ de largo, de 3-3.5 $\mathrm{mm}$ de ancho.

Distribución y ecología. Hasta donde se conoce, Comarostaphylis macvaughii es endémico de una de las partes más altas de la Sierra de Tapalpa, al S de Jalisco, situada a los $20^{\circ} 00^{\prime}$ de latitud N y $104^{\circ} 12^{\prime}$ de longitud W. Se encuentra en una ladera de exposición SE, con pendiente de $15^{\circ}$, en el claro de un bosque de Pinus lumholtzii asociado a Quercus spp., Arbutus glandulosa, Comarostaphylis glaucescens, Vaccinium stenophyllum y Arctostaphylos pungens. El taxon es muy abundante localmente, al extremo de cubrir una extensión considerable y en la época de mayor floración en los meses de junio a agosto, el área está cubierta de flores rosadas.

Material examinado: Jalisco, municipio de Tapalpa, microondas Brizuela, km 10 camino Tapalpa-Chiquilistlán, alt. $2300 \mathrm{~m}$, arbustito rizomatoso de $10-15 \mathrm{~cm}$ de alto, de flores rosadas, muy abundante en bosque de Pinus lumholtzii, Quercus castanea, Q. obtusata, Arbutus glandulosa, Comarostaphylis glaucescens, Arctostaphylos pungens, Panicum bulbosum, Rhynchosporasp., 2. VIII. 1986, González Villarreal2596 al 2598(IBUG), ejemplares con flores y frutos; ibid., 15.X.1988. González Villarreal 3448, 3449 (IBUG), ejemplares estériles.

Comarostaphylis macvaughii fue descrito por Diggs (1988) como una subespecie de $C$. discolor, pero los miembros de estaúltima especie se caracterizan por ser arbustos de (0.50-) 1-5 m de alto, con ramillas glabras a pubescentes o tomentosas o con denso tomento ferrugíneo y tricomas glandulares, con hojas lanceolado-elípticas a oblongas o elípticas a elíptico-lanceoladas, de $4-20 \mathrm{~cm}$ de largo y de $1.1-4.5 \mathrm{~cm}$ de ancho, de margen aserrulado, aserrado o dentado-aserrado y con inflorescencias generalmente en panículas de 5-17 cm de largo. En cambio, C. macvaughii se distingue por ser un arbustito cespitoso de (6-) 10-15 cm 
de alto, carente de pubescencia, con hojas lineares a linear-lanceoladas, enteras, de $1-4 \mathrm{~cm}$ de largo, de 1-5 mm de ancho, las más pequeñas conocidas para el género y por sus inflorescencias en racimos extremadamente reducidos, de $1.5-2.5 \mathrm{~cm}$ de largo, incluso algunas veces se presentan flores solitarias (véase Cuadro 1).

Cuadro 1. Tabla comparativa de algunos caracteres morfológicos de Comarostaphylis macvaughii y de las subespecies de $C$. discolor

\begin{tabular}{|c|c|c|c|c|}
\hline Características & C. macvaughii & $\begin{array}{l}\text { C. discolor } \\
\text { ssp. discolor }\end{array}$ & $\begin{array}{l}\text { C. discolor ssp. } \\
\text { manantlanensis }\end{array}$ & $\begin{array}{l}\text { C. discolor } \\
\text { ssp. rupestris }\end{array}$ \\
\hline Hábito & $\begin{array}{c}\text { arbustito cespitoso de } \\
\text { (6-) } 10-15(-25) \mathrm{cm} \\
\text { de alto }\end{array}$ & $\begin{array}{c}\text { arbusto de } \\
\text { (0.50-) } 1-3 \mathrm{~m} \text { de alto }\end{array}$ & $\begin{array}{l}\text { arbusto de } \\
2-3 \mathrm{~m} \text { de alto }\end{array}$ & $\begin{array}{l}\text { arbusto de } 1-5 \mathrm{~m} \\
\text { de alto }\end{array}$ \\
\hline Ramillas & glabras & $\begin{array}{c}\text { glabras a } \\
\text { pubescentes }\end{array}$ & $\begin{array}{l}\text { con denso tomento } \\
\text { ferrugíneo y con } \\
\text { tricomas glandulares }\end{array}$ & $\begin{array}{l}\text { pubescentes o } \\
\text { tomentosas }\end{array}$ \\
\hline $\begin{array}{l}\text { Forma de } \\
\text { la hoja }\end{array}$ & $\begin{array}{l}\text { linear a linear- } \\
\text { lanceolada }\end{array}$ & $\begin{array}{l}\text { lanceolado-elíptica } \\
\text { a oblonga }\end{array}$ & $\begin{array}{l}\text { elíptica a elíptico- } \\
\quad \text { lanceolada }\end{array}$ & $\begin{array}{c}\text { lanceolado-eliptica } \\
\text { a oblonga }\end{array}$ \\
\hline $\begin{array}{ll}\text { Hoja } & \text { (largo) } \\
& \text { (ancho) }\end{array}$ & $\begin{array}{l}(1-1) 1.5-2(-4) \mathrm{cm} \\
(1-1.5-2(-5) \mathrm{mm}\end{array}$ & $\begin{array}{l}(4-) 7-9(-15.7) \mathrm{cm} \\
1.5-2.5(-3.6) \mathrm{cm}\end{array}$ & $\begin{array}{c}(6-) 8-17(-20) \mathrm{cm} \\
(1.1-) 2.5-3(-4.5) \mathrm{cm}\end{array}$ & $\begin{array}{l}7-16 \mathrm{~cm} \\
1.5-3.5 \mathrm{~cm}\end{array}$ \\
\hline $\begin{array}{c}\text { Margen de } \\
\text { la hoja }\end{array}$ & entero & $\begin{array}{l}\text { aserrulado, aserrado } \\
\text { o dentado-aserrado, } \\
\text { rara vez entero }\end{array}$ & aserrado & $\begin{array}{l}\text { aserrado, rara } \\
\text { vez entero }\end{array}$ \\
\hline $\begin{array}{l}\text { Inflorescencia } \\
\quad \text { (largo) }\end{array}$ & $\begin{array}{l}\text { racimo de } \\
1.5-2(-2.5) \mathrm{cm}\end{array}$ & $\begin{array}{l}\text { racimo o panicula } \\
\text { de } 5-6(-10.5) \mathrm{cm}\end{array}$ & $\begin{array}{c}\text { panicula de } \\
(4-5-8.5(-17) \mathrm{cm}\end{array}$ & $\begin{array}{l}\text { panicula de } \\
5-8 \mathrm{~cm}\end{array}$ \\
\hline $\begin{array}{l}\text { Raquis de la } \\
\text { inflorescencia }\end{array}$ & glabro & $\begin{array}{c}\text { glabro o con tricomas } \\
\text { glandulares }\end{array}$ & $\begin{array}{l}\text { con tricomas } \\
\text { glandulares }\end{array}$ & $\begin{array}{l}\text { puberulento a } \\
\text { tomentoso con trico- } \\
\text { mas eglandulares }\end{array}$ \\
\hline Corola & rosada & $\begin{array}{c}\text { blanca a } \\
\text { blanco-rosada }\end{array}$ & $\begin{array}{l}\text { blanco-amarillenta a } \\
\text { ligeramente verdosa }\end{array}$ & blanco-verdosa \\
\hline Ovario & glabro & glabro & $\begin{array}{c}\text { piloso, a veces } \\
\text { lanoso }\end{array}$ & glabro \\
\hline
\end{tabular}

Se ha comprobado en las visitas al campo que los individuos de ambos taxa, que habitan en la zona de Tapalpa, crecen en la misma área pero no se mezclan. Los arbustos de $C$. discolor son abundantes pero se encuentran aislados entre sí y en su mayoría son de cerca de $1 \mathrm{~m}$, aunque algunos alcanzan hasta $3 \mathrm{~m}$ de alto. Los más pequeños a menudo presentan reducción en el tamaño de las hojas, pero mantienen el carácter de borde dentado-aserrado. En cambio, C. macvaughii es un arbustito cespitoso de $10-15 \mathrm{~cm}$, los individuos más vigorosos apenas son de $25 \mathrm{~cm}$ de alto; las hojas son de menor tamaño que las de $C$. discolor subsp. discolor y el borde es entero. 
Diggs (1988) refiere que en su visita a Tapalpa encontró que el gradiente en el tamaño de las hojas de $C$. discolor subsp. discolor es muy amplio, existiendo hojas muy pequeñas (aún en la misma planta), por lo que decide relacionar el nuevo taxon con la subsp. discolor; por otra parte, también indica que "las plantas habitan como individuos aislados... se distinguen morfologicamente por su desarrollo extremadamente bajo (de ca. de $1 \mathrm{~m}$ de alto, pero con frecuencia florecen cuando tienen ca. de $10 \mathrm{~cm}$ de alto".

Estoy de acuerdo con Diggs (1981) en su identificación original como $C$. discolorsubsp. discolordel ejemplar Villarreal de Puga 1697(ENCB, IBUG), colectado en el Cerro El Fraile, ca. de Tapalpa, 2400 m, 26.V.1968, (fl). Sin embargo, después de su visita en el mes de junio de 1986 a las montañas cercanas a Tapalpa y en función de sus observaciones y colectas realizadas, el mencionado autor decidió considerarlo como $C$. discolor subsp. macvaughii (Diggs, 1988).

Estos hechos me llevan a concluir que, en el transcurso de sus exploraciones de la zona de Tapalpa Diggs probablemente no encontró la localidad tipo de $C$. macvaughii ni ha visto plantas vivas de este taxon y al describirlo utilizó material mixto que incluyó ejemplares de $C$. discolor subsp. discolor. Puesto que el protólogo original está basado en especímenes que pertenecen a dos taxa distintos, se consideró pertinente presentar en este trabajo una descripción emanada exclusivamente de topotipos.

Comarostaphylis macvaughii no parece estar morfológicamente muy relacionado con C. discolor subsp. discolor y tal vez muestra más afinidad con C. polifolia (H. B. K. ) Zucc. ex Klotz. subsp. minor (Small) Diggs. Las características comunes con este último taxon son: arbustos carentes de tricomas glandulares, hojas enteras cortamente pecioladas, filamentos vilosos y ovario glabro. Las diferencias se muestran en el Cuadro 2.

Cuadro 2. Caracteres distintivos entre Comarostaphylis macvaughii y $C$. polifolia subsp. minor

\begin{tabular}{|c|c|c|}
\hline Caracter & C. macvaughii & C. polifolia subsp. minor \\
\hline Altura & $(6-) 10-15(-25) \mathrm{cm}$ & $1-4 \mathrm{~m}$ \\
\hline Forma de la hoja & linear a linear-lanceolada & oblanceolada a eliptica \\
\hline Apice de la hoja & agudo a acuminado, mucronado & obtuso a redondeado \\
\hline Envés de la hoja & glabro & puberulento \\
\hline Hoja (largo) & ancho) & $(1-) 1.5-2(-4) \mathrm{cm}$ \\
\hline Inflorescencia & racimo con raquis glabro & $\begin{array}{c}2.2-6.6 \mathrm{~cm} \\
0.3-1.9 \mathrm{~cm}\end{array}$ \\
\hline Distribución & Jalisco & $\begin{array}{c}\text { a pubescente o ligeramente } \\
\text { canescente }\end{array}$ \\
\hline Hábitat & bosque de pino y encino & $\begin{array}{c}\text { Nuevo León, Tamaulipas } \\
\text { San Luis Potosi }\end{array}$ \\
\hline
\end{tabular}


Por las notables características anteriormente mencionadas para C. macvaughii, se infiere que la categoría de subespecie no le fue correctamente asignada, y se hace esta nota con la intención de corregir tal situación, proponiendo un nuevo status.

\section{AGRADECIMIENTOS}

Mi más cumplido agradecimiento al Dr. Jerzy Rzedowski por su guía constante y consejos desde siempre. Gracias al Dr. James Luteyn del New York Botanical Garden por su amable crítica. A Ma. del Refugio Vázquez Velasco autora de la ilustración.

\section{LITERATURA CITADA}

Diggs, G. M. Jr. 1981. Systematic studies in the Arbuteae (Ericaceae, Vaccinioideae) including a revision of the genus Comarostaphylis. Tesis doctoral. University of Wisconsin-Madison. Madison. 343 pp.

Diggs, G. M. Jr. 1986. A new subspecies and new combinations in Comarostaphylis (Ericaceae). Brittonia 38(4): 344-351.

Diggs, G. M. Jr. 1988. New subspecies in Comarostaphylis discolor (Ericaceae, Arbuteae) from Jalisco, México. Bull. Torrey Bot. Club 115(3): 203-208. 